\title{
MYH rs3219476 and rs3219472 polymorphisms and risk of cholangiocarcinoma
}

\author{
SI-HONG YOU ${ }^{1}$, XIANG WANG $^{1}$, SHU HUANG ${ }^{1}$, MIN WANG $^{1}$, GUO-ZHONG JI ${ }^{1}$, \\ JIN-RONG XIA ${ }^{2}$ and ZHI-NING FAN ${ }^{1}$ \\ ${ }^{1}$ Medical Center for Digestive Diseases, Second Affiliated Hospital of Nanjing Medical University, Nanjing 210011; \\ ${ }^{2}$ Department of Gastroenterology, ZhongDa Hospital, Southeast University, Nanjing 210009, P.R. China
}

Received July 2, 2012; Accepted October 30, 2012

DOI: $10.3892 / \mathrm{mmr} .2012 .1175$

\begin{abstract}
Cholangiocarcinoma (CCA) is a rare but devastating malignancy. Up to $90 \%$ of patients presenting with CCA have no identifiable risk factors. The base excision repair (BER) pathway has a principal role in the repair of mutations caused by oxidized or reduced bases. The MutY homolog (MUTYH, $\mathrm{MYH}$ ) is one of the key proteins in the BER pathway, but the role of MYH in the tumorigenesis of CCA is largely unknown. In this study, we investigated the influence of MYH rs3219476 and rs3219472 polymorphisms on CCA incidence. MYH genotypes were detected using a polymerase chain reactionrestriction fragment length polymorphism (PCR-RFLP) technique. We found that for rs3219472, compared with subjects carrying the MYH G/G genotype, those with the A/A genotype had a 2.816-fold higher risk of CCA [odds ratio $(\mathrm{OR})=2.816,95 \%$ confidence interval $(\mathrm{CI})=0.992-7.999$, $\mathrm{P}=0.047)$. For rs3219476, compared with subjects carrying the MYH T/T genotype, those with the T/G genotype had a reduced risk of $\mathrm{CCA}(\mathrm{OR}=0.359,95 \% \mathrm{CI}=0.17-0.758, \mathrm{P}=0.006)$. Our findings suggest that since significantly increased CCA risk was found in individuals with a homozygous variant genotype for rs3219472, it may be a biomarker for screening individuals at high risk of developing the disease.
\end{abstract}

\section{Introduction}

Cholangiocarcinoma (CCA), originating from the epithelial cells lining the biliary duct, is a rare but devastating malignancy $(1,2)$. CCAs are clinically silent in the majority of cases (3). Radical resection of the tumor is applicable only in a

Correspondence to: Professor Zhi-Ning Fan, Medical Center for Digestive Diseases, Second Affiliated Hospital of Nanjing Medical University, 121 Jiang Jia Yuan, Nanjing 210011, P.R. China

E-mail: fanzhining@hotmail.com

Professor Jin-Rong Xia, Department of Gastroenterology, ZhongDa Hospital, Southeast University, 87 Ding Jia Qiao Road, Nanjing 210009, P.R. China

E-mail: xjr049540@163.com

Key words: cholangiocarcinoma, MutY homolog, polymorphism minority of patients due to late clinical presentation and diagnosis (4). Moreover, the recurrence rate following resection is extremely high (5). Although advanced CCAs may respond to chemotherapy, there are no standard treatments for the palliation of advanced disease (3). The overall survival rate of the disease is poor (6).

Although a number of risk factors have been identified, including primary sclerosing cholangitis (PSC), infestation with liver flukes, toxic compounds, congenital disorders, hepatolithiasis, viral hepatitis, alcohol abuse, smoking, obesity and diabetes $(4,7)$, only a minority of CCA patients have known risk factors. Up to $90 \%$ of patients presenting with CCA have no identifiable risk factors $(4,6)$.

CCA, in common with certain other cancers, is caused by gene-environment interactions. Carcinogens induce tumorigenesis in genetically susceptible individuals $(8,9)$. Genomic DNA is continuously attacked by a large number of agents that damage DNA. However, cancers only occur in a small proportion of people since DNA damage is normally repaired by genome surveillance mechanisms and DNA repair pathways, including homologous recombination (HR), base excision repair (BER), nucleotide excision repair (NER) and mismatch repair (MMR) (10). Polymorphisms of DNA repair genes may affect the quantity and activity of the resulting protein and the DNA repair capacity. The possession of specific alleles of polymorphic genes may increase susceptibility to cancers and facilitate cancer development in normal or exposed individuals (11).

The BER pathway has a principal role in the repair of mutations caused by oxidized or reduced bases. Human oxoguanine glycosylase 1 (hOGG1), MutY homolog (MUTYH, MYH), apurinic/apyrimidinic endonuclease-1 (APEX1, APE1) and X-ray cross-complementing group 1 (XRCC1) are key proteins in the BER pathway (12-14). We have previously analyzed hOGG1 Ser326Cys and XRCC1 codon 194 and 399 polymorphisms in CCAs and did not find an association between polymorphisms of these genes and susceptibility to CCA $(15,16)$.

MYH is involved in the repair of mutations caused by oxidized bases $(13,14)$. Harmful environmental agents may cause the production of reactive oxygen species (ROS), including hydroxyl radicals, in the body (14). Oxidative DNA damage occurs when the production of ROS exceeds the anti- 
oxidant capacity of the cell (17). 8-Hydroxy-2'-deoxyguanosine (8-oxoG) is one of the most abundant and stable products of oxidative DNA damage and may cause G:C to T:A transversions $(14,17)$. The BER pathway is responsible for repairing 8-oxoG lesions. MYH is a DNA glycosylase that removes adenine paired with 8-oxoG (13). Biallelic loss of MYH is associated with somatic $\mathrm{G}: \mathrm{C}$ to $\mathrm{T}: \mathrm{A}$ transversions in the adenomatous polyposis coli (APC) gene and is responsible for polyp formation and colorectal cancer (18). However, the role of MYH in the tumorigenesis of CCA is largely unknown. It has been reported that one SNP in the MYH gene (rs3219476) was associated with an increased risk of treatment-related mortality (TRM) in recipients who took alkylating agents with or without ionizing radiation for pretransplant conditioning regimens and underwent allogeneic hematopoietic cell transplant (HCT) for the treatment of hematologic malignancies (19). However, the relationship between MYH rs3219476 polymorphism and the risk of CCA has not been reported. In this study, we evaluated the influence of polymorphisms of two adjacent SNPs (rs3219476 and rs3219472) in the MYH gene on the risk of CCA in a Chinese population.

\section{Materials and methods}

Subjects. A hospital-based case-control study of CCA was conducted in the Second Affiliated Hospital of Nanjing Medical University between May 2007 and June 2010. Written informed consent was obtained from all participants and the study was approved by the Ethics Committee of the Hospital. The cases were 59 CCA patients aged from 27 to 86 years with an average of $64.7 \pm 15.5$ years. Among the patients, 37 cases were male and 22 were female. The patients were diagnosed with CCA according to clinical presentations and imaging, including computerized tomography (CT), magnetic resonance imaging (MRI), magnetic resonance cholangiopancreatography (MRCP) and endoscopic retrograde cholangiopancreatography (ERCP) (20). Ten cases of CCA diagnosis were confirmed by histology. None of the cases had a history of any cancer other than CCA. A total of 100 controls with no current or previous diagnosis of cancer or other major diseases were recruited. Among the controls, 59 were male, 41 were female, and the average age was $61.7 \pm 10.2$ years. All subjects were unrelated Han Chinese living in Jiangsu province.

Blood samples and DNA isolation. Venous blood $(3 \mathrm{ml})$ was collected from each of the CCA patients or controls. After collection, the whole blood was immediately stored at $-80^{\circ} \mathrm{C}$ until use. Genomic DNA was extracted from the blood samples by a routine phenol-chloroform method.

Genotyping of MYH rs3219476 and rs3219472 polymorphisms. MYH genotypes were detected using a polymerase chain reaction-restriction fragment length polymorphism (PCR-RFLP) technique. MYH rs3219476 and rs3219472 were amplified to form undigested fragments of 363 and $176 \mathrm{bp}$, respectively, using primers designed by ourselves with a web-based PCR-RFLP designing tool, SNP PCR-RFLP assay design at http://bioapp.psych.uic.edu/SNP_cutter.htm. The primers used were as follows: forward 5'-GGA AGG AAG

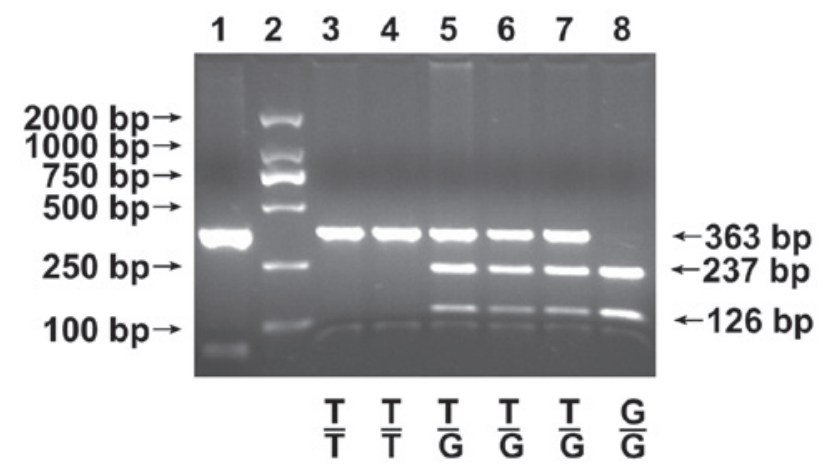

Figure 1. Representative results for MutY homolog (MYH) rs3219476 polymorphisms by polymerase chain reaction-restriction fragment length polymorphism (PCR-RFLP) methods. Lane 1: PCR product; lane 2: markers; lanes 3 and 4: the wild-type genotype T/T had no BtsCI site and was characterized by a 363 bp fragment; lanes 5-7: the heterozygous genotype T/G was characterized by 363, 237 and 126 bp fragments; lane 8: the variant genotype $\mathrm{G} / \mathrm{G}$ was characterized by 237 and 126 bp fragments.

TGA CGT GAT CTG G-3' and reverse 5'-TGG TGG TCT CTT CAC ATG GAC A-3' for rs3219476; forward 5'-GTA AGA GCT ACA AGG CAG-3' and reverse 5'-GCT TAA ACA CCT CAA GTG-3' for rs3219472. Briefly, 50 ng genomic DNA was amplified in a volume of $50 \mu 1$ containing $1 \mathrm{X}$ PCR buffer, $1.5 \mathrm{mM} \mathrm{MgCl}{ }_{2}, 0.2 \mathrm{mM}$ of each dNTP, $0.5 \mu \mathrm{M}$ of each primer and 1.25 units Taq DNA Polymerase (Takara Biotechnology, Dalian, China). The PCR conditions were a 5 min denaturation step at $94^{\circ} \mathrm{C}$; followed by 40 cycles of $94^{\circ} \mathrm{C}$ for $30 \mathrm{sec}, 60^{\circ} \mathrm{C}$ for $30 \mathrm{sec}$ and $72^{\circ} \mathrm{C}$ for $30 \mathrm{sec}$; and a final extension at $72^{\circ} \mathrm{C}$ for $10 \mathrm{~min}$. After amplification, $8 \mu \mathrm{l}$ PCR products were digested with $B t s \mathrm{CI}$ at $50^{\circ} \mathrm{C}$ or $T s p 509 \mathrm{I}$ at $65^{\circ} \mathrm{C}$ for the genotyping of rs3219476 or rs3219472, respectively, for $4 \mathrm{~h}$ in a final volume of $20 \mu \mathrm{l}$ containing $1 \mathrm{X}$ NE buffer and $0.5 \mu 1$ (2.5 units) restriction enzyme (New England Biolabs, Beverly, MA, USA). The digested fragments were separated by electrophoresis on $2 \%(\mathrm{w} / \mathrm{v})$ agarose gel and visualized by ethidium bromide staining using a DL2000 DNA Marker (Takara BioTechnology). All experiments included positive and negative controls. For rs3219476, the wild-type genotype T/T had no BtsCI site and was characterized by a 363 bp fragment on the gel; the variant genotype $\mathrm{G} / \mathrm{G}$ was characterized by 237 and $126 \mathrm{bp}$ fragments; and the heterozygous genotype T/G was characterized by 363,237 and 126 bp fragments (Fig. 1). In the genotyping of rs3219472, the complete digestion of PCR products produced $176 \mathrm{bp}$ fragments for the $\mathrm{G}$ allele and $128 \mathrm{bp}$ and $48 \mathrm{bp}$ fragments for the A allele (the $48 \mathrm{bp}$ fragment was too small to resolve accurately; Fig. 2). DNA sequencing was performed to confirm the genotypes in certain cases (Figs. 3 and 4).

Statistical analysis. Mean and standard deviations are presented in cases of continuous variables. Differences between the means of the two continuous variables were evaluated by the Student's t test. The Hardy-Weinberg equilibrium was tested using a web-based program, OEGE (Online Encyclopedia for Genetic Epidemiology) at http://www.oege. org/software/hwe-mr-calc.shtml, to compare the observed genotype frequencies with the expected frequencies among the control subjects. The Chi-square test was used to compare 
$\begin{array}{llllllllllll}1 & 2 & 3 & 4 & 5 & 6 & 7 & 8 & 9 & 10 & 11\end{array}$

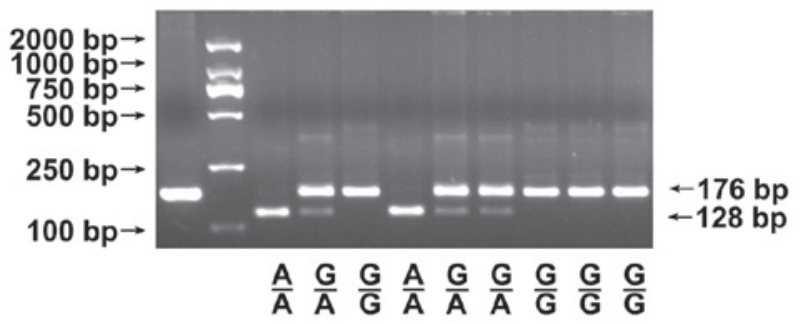

Figure 2. Representative results for the MutY homolog (MYH) rs3219472 polymorphisms by polymerase chain reaction-restriction fragment length polymorphism (PCR-RFLP) methods. Lane 1: PCR product; lane 2: markers; lanes 3 and 6: the variant genotype A/A was characterized by a 128 bp fragment; lanes 5 and 9-11: the wild-type genotype G/G had no Tsp509I site and was characterized by a 176 bp fragment; lanes 4,7 and 8 : the heterozygous genotype G/A was characterized by 176 and 128 bp fragments.

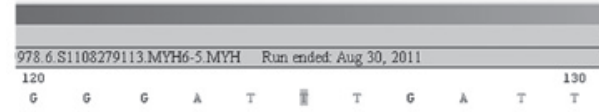

$\mathrm{T} / \mathrm{T}$

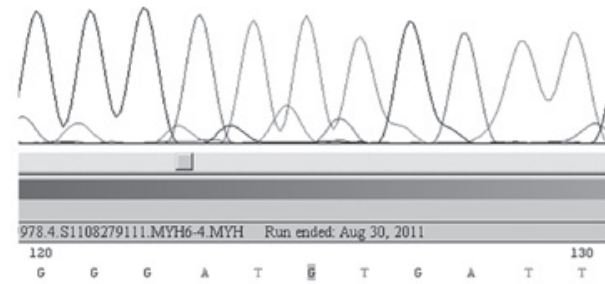

T/G
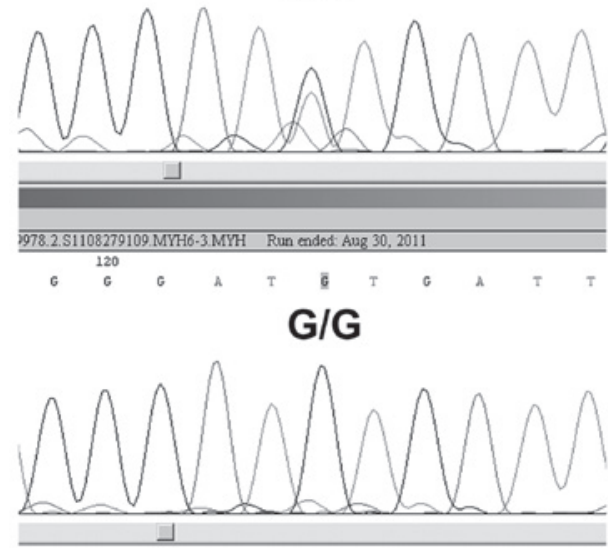

Figure 3. DNA sequencing and genotyping for MutY homolog (MYH) rs3219476 polymorphisms. T/T, T/G and G/G represent the wild-type, heterozygote and variant, respectively.

the gender distribution and test the association between the genotypes and alleles in relation to the cases and controls. $\mathrm{P}<0.05$ was considered to indicate a statistically significant result. The odds ratio (OR) and $95 \%$ confidence interval $(95 \%$ CI) were calculated to estimate the associations between the genotypes of MYH and the risk of CCA. Statistical analyses were carried out using SPSS version 11.0 software (SPSS, Chicago, IL, USA) and Microsoft Excel 2003 (Microsoft Corporation, Seattle, USA).

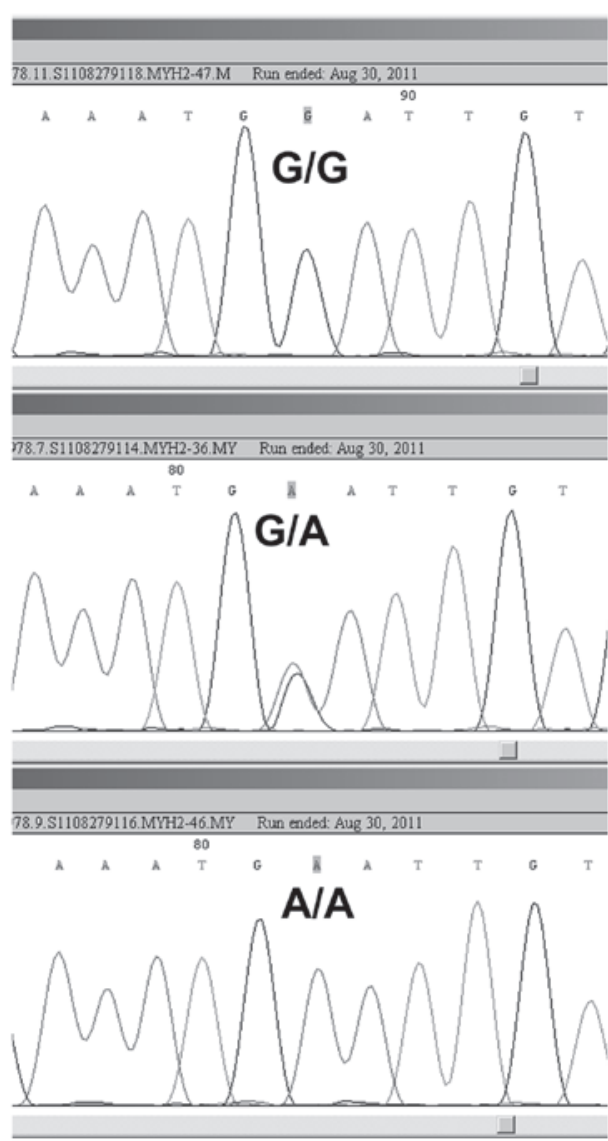

Figure 4. DNA sequencing and genotyping for MutY homolog $(\mathrm{MYH})$ rs3219472 polymorphisms. G/G, G/A and A/A represent the wild-type, heterozygote and variant, respectively.

\section{Results}

The case and control groups were not statistically different with respect to age $(\mathrm{t}=1.335, \mathrm{P}=0.185)$ and gender $\left(\chi^{2}=0.214\right.$, $\mathrm{P}=0.644$ ). The frequencies of the MYH rs3219472 genotypes associated with CCA are shown in Table I. For rs3219472, the genotype frequencies of $\mathrm{G} / \mathrm{G}, \mathrm{G} / \mathrm{A}$ and $\mathrm{A} / \mathrm{A}$ were $47.5,32.2$ and $20.3 \%$, respectively, in the CCA cases compared with $46.0,47.0$ and $7.0 \%$, respectively, in the controls. The genotype distribution in the controls was within the Hardy-Weinberg equilibrium $\left(\chi^{2}=1.18, \mathrm{P}>0.05\right)$. The frequency of the MYH rs3219472 A allele was $36.4 \%$ in the CCA group and $30.5 \%$ in the control group. Statistically significant differences were observed in the genotype frequencies of the MYH rs3219472 polymorphism between the control group and the patients with CCA $\left(\chi^{2}=7.499, \mathrm{P}=0.024\right)$, but no statistically significant differences were identified in the allele frequencies of the MYH rs3219472 polymorphism between the two groups $\left(\chi^{2}=1.190\right.$, $\mathrm{P}=0.275)$. Compared with subjects carrying the $\mathrm{MYH} \mathrm{G/G}$ genotype, those with the A/A genotype had a 2.816-fold higher risk of $\mathrm{CCA}(\mathrm{OR}=2.816,95 \% \mathrm{CI}=0.992-7.999, \mathrm{P}=0.047)$.

As shown in Table II, for rs3219476, the genotype frequencies of $\mathrm{T} / \mathrm{T}, \mathrm{T} / \mathrm{G}$ and $\mathrm{G} / \mathrm{G}$ were $42.4,33.9$ and $23.7 \%$, respectively, in the CCA cases compared with 26.0, 58.0 and $16.0 \%$, respectively, in the controls. The genotype distribution in the controls was within the Hardy-Weinberg equilibrium 
Table I. Distribution of allele and genotype frequencies of MutY homolog (MYH) rs3219472 polymorphism in cholangiocarcinoma (CCA) patients and healthy controls.

\begin{tabular}{lcccr}
\hline & CCA, n $(\%)$ & Controls, $n(\%)$ & OR (95\% CI) & P-value \\
\hline Genotypes & & & & - \\
G/G & $28(47.5)$ & $46(46.0)$ & $1(-)$ & 0.258 \\
G/A & $19(32.2)$ & $47(47.0)$ & $0.664(0.326-1.351)$ & 0.047 \\
A/A & $12(20.3)$ & $7(7.0)$ & $2.816(0.992-7.999)$ & 0.859 \\
G/A+A/A & $31(52.5)$ & $54(54.0)$ & $0.943(0.495-1.797)$ & - \\
Alleles & & & & $1(-)$ \\
G & $75(63.6)$ & $139(69.5)$ & $1.306(0.808-2.113)$ & 0.275 \\
A & $43(36.4)$ & $61(30.5)$ & & \\
\hline
\end{tabular}

Table II. Distribution of allele and genotype frequencies of MutY homolog (MYH) rs3219476 polymorphism in cholangiocarcinoma (CCA) patients and healthy controls.

\begin{tabular}{lcccr}
\hline & CCA, $n(\%)$ & Controls, $n(\%)$ & OR (95\% CI) & P-value \\
\hline Genotypes & & & & - \\
T/T & $25(42.4)$ & $26(26.0)$ & $0.359(0.17-0.758)$ & 0.006 \\
T/G & $20(33.9)$ & $58(58.0)$ & $0.91(0.369-2.246)$ & 0.838 \\
G/G & $14(23.7)$ & $16(16.0)$ & $0.478(0.241-0.946)$ & 0.033 \\
T/G+G/G & $34(57.6)$ & $74(74.0)$ & & - \\
Alleles & & & $1(-)$ & 0.453 \\
T & $70(59.3)$ & $110(55.0)$ & $0.838(0.529-1.329)$ & \\
G & $48(40.7)$ & $90(45.0)$ & & \\
\hline
\end{tabular}

$\left(\chi^{2}=2.95, \mathrm{P}>0.05\right)$. The frequency of the MYH rs $3219476 \mathrm{G}$ allele was $40.7 \%$ in the CCA group and $45.0 \%$ in the control group. Statistically significant differences were observed in the genotype frequencies of the MYH rs3219476 polymorphism between the control group and the patients with CCA $\left(\chi^{2}=8.670, P=0.013\right)$, but no statistically significant differences were identified in the allele frequencies of the MYH rs3219476 polymorphism between the two groups $\left(\chi^{2}=0.564, \mathrm{P}=0.453\right)$. Compared with subjects carrying the MYH T/T genotype, those with the $\mathrm{T} / \mathrm{G}$ genotype had a reduced risk of CCA $(\mathrm{OR}=0.359,95 \% \mathrm{CI}=0.17-0.758, \mathrm{P}=0.006)$.

\section{Discussion}

The MYH gene excises adenine bases from the DNA backbone at sites where adenine is inappropriately paired with guanine, cytosine or 8-oxoG (19). Although multiple mutations (Y165C, G382D, R260Q and H434D) or single nucleotide polymorphisms (rs3219484 and rs3219489) have been identified in the MYH gene $(21,22)$, no associations have been identified between these variants and the susceptibility to CCA $(14,22)$. Furthermore, the Y165C and G382D mutations are rarely detected in Chinese Han populations $(23,24)$. The two SNPs analyzed in our study, rs3219472 and rs3219476, are located in the intron region near the 5'-UTR of the MYH gene. In this study, significantly increased CCA risk was found in individuals with the homozygous variant genotype for rs3219472, but no increased risk was identified in those with the homozygous variant genotype for rs3219476. Furthermore, a reduced risk of CCA was found in those with heterozygous genotype for rs3219476.

The minor allele frequency (MAF) for rs3219476 (0.450) in the controls in the current study were comparable to those in the HapMap-HCB and CHB_GENO_PANEL databases (MAF $=0.444$ and 0.443 , respectively), which are based on the Chinese Han population. However, the MAF for rs3219472 (0.305) in the controls in the current study was slightly different from that in the HAPMAP-CHB database (MAF=0.363), which is also based on the Chinese Han population, reflecting sampling errors and regional differences between the different data. It has been reported that MYH rs3219476 polymorphism was associated with 1-year TRM in HCT patients who accepted ionizing radiation and alkylating agents that damaged DNA (19). However, the effect of MYH rs3219476 and rs3219472 polymorphism on the risk of CCA is unknown. To our knowledge, this is the first study to evaluate the ability of MYH rs3219476 and rs3219472 polymorphisms to predict the susceptibility to CCA. Our findings suggest that male Han Chinese with the MYH A/A genotype in rs3219472 have a higher susceptibility to CCA. It may be a biomarker for screening individuals at high risk of developing the disease.

However, our data may be biased by the relatively small number of subjects and requires validation in larger samples. 
As CCA is a rare disease (3), multiple center cooperation would be necessary to recruit enough CCA patients for investigation. Further studies of the precise mechanisms by which MYH polymorphism affects the risk of CCA are merited.

\section{Acknowledgements}

This study was supported by the Natural Science Foundation of Jiangsu Province, No. BK2007260.

\section{References}

1. Isomoto H: Epigenetic alterations associated with cholangiocarcinoma (Review). Oncol Rep 22: 227-232, 2009.

2. Petrowsky H and Hong JC: Current surgical management of hilar and intrahepatic cholangiocarcinoma: the role of resection and orthotopic liver transplantation. Transplant Proc 41: 4023-4035, 2009.

3. Valle J, Wasan H, Palmer DH, et al: Cisplatin plus gemcitabine versus gemcitabine for biliary tract cancer. N Engl J Med 362: $1273-1281,2010$

4. Gatto M, Bragazzi MC, Semeraro R, et al: Cholangiocarcinoma: update and future perspectives. Dig Liver Dis 42: 253-260, 2010.

5. Shen FZ, Zhang BY, Feng YJ, et al: Current research in perineural invasion of cholangiocarcinoma. J Exp Clin Cancer Res 29: 24, 2010

6. Meza-Junco J, Montano-Loza AJ, Ma M, Wong W, Sawyer MB and Bain VG: Cholangiocarcinoma: has there been any progress? Can J Gastroenterol 24: 52-57, 2010.

7. Ustundag Y and Bayraktar Y: Cholangiocarcinoma: a compact review of the literature. World J Gastroenterol 14: 6458-6466, 2008.

8. Shields PG and Harris CC: Cancer risk and low-penetrance susceptibility genes in gene-environment interactions. J Clin Oncol 18: 2309-2315, 2000

9. Galvan A, Ioannidis JP and Dragani TA: Beyond genome-wide association studies: genetic heterogeneity and individual predisposition to cancer. Trends Genet 26: 132-141, 2010.

10. Martin SA, Hewish M, Lord CJ and Ashworth A: Genomic instability and the selection of treatments for cancer. J Pathol 220: 281-289, 2010.
11. Jiang J, Zhang X, Yang H and Wang W: Polymorphisms of DNA repair genes: ADPRT, XRCC1, and XPD and cancer risk in genetic epidemiology. Methods Mol Biol 471: 305-333, 2009.

12. David SS, O'Shea VL and Kundu S: Base-excision repair of oxidative DNA damage. Nature 447: 941-950, 2007.

13. Kasahara M, Osawa K, Yoshida K, et al: Association of MUTYH Gln324His and APEX1 Asp148Glu with colorectal cancer and smoking in a Japanese population. J Exp Clin Cancer Res 27: 49, 2008.

14. Baudhuin LM, Roberts LR, Enders FT, et al: MYH Y165C and G382D mutations in hepatocellular carcinoma and cholangiocarcinoma patients. J Cancer Res Clin Oncol 132: 159-162, 2006.

15. Wang X, You SH, He W, et al: hOGG1 Ser326Cys polymorphisms and genetic susceptibility of cholangiocarcinomas. Journal of Medical Postgraduates 23: 1156-1159, 2010 (In Chinese).

16. You SH, Wang X, Huang S, Gao Y, Xia JR and Fan ZN: XRCC1 polymorphisms and genetic susceptibility of cholangiocarcinomas. Chinese Journal of Clinical Research 23: 1068-1072, 2010 (In Chinese).

17. Tsukino H, Hanaoka T, Otani T, et al: hOGG1 Ser326Cys polymorphism, interaction with environmental exposures, and gastric cancer risk in Japanese populations. Cancer Sci 95: 977-983, 2004.

18. Jones S, Emmerson P, Maynard J, et al: Biallelic germline mutations in MYH predispose to multiple colorectal adenoma and somatic G:C->T:A mutations. Hum Mol Genet 11: 2961-2967, 2002.

19. Thyagarajan B, Lindgren B, Basu S, et al: Association between genetic variants in the base excision repair pathway and outcomes after hematopoietic cell transplantations. Biol Blood Marrow Transplant 16: 1084-1089, 2010.

20. Van Beers BE: Diagnosis of cholangiocarcinoma. HPB (Oxford) 10: 87-93, 2008

21. Sieber OM, Lipton L, Crabtree M, et al: Multiple colorectal adenomas, classic adenomatous polyposis, and germ-line mutations in MYH. N Engl J Med 348: 791-799, 2003.

22. Forsbring M, Vik ES, Dalhus B, et al: Catalytically impaired hMYH and NEIL1 mutant proteins identified in patients with primary sclerosing cholangitis and cholangiocarcinoma. Carcinogenesis 30: 1147-1154, 2009

23. Yang L, Zhou M, Zhu M, et al: Variation analysis of the MYH gene associated with sporadic colorectal cancer. Carcinogenesis, Teratogenesis and Mutagenesis 21: 435-438, 2009 (In Chinese).

24. Yang L, Zhu M, Chen SQ, et al: Research on MUTYH gene polymorphism associated with colorectal cancer. Shandong Medical Journal 49: 3-5, 2009 (In Chinese). 